\title{
Stigma and Empathy: Sex Workers as Educators of Medical Students
}

\author{
Richard Balon ${ }^{1}$ - Glendon R. Tait ${ }^{2} \cdot$ John H. Coverdale ${ }^{3} \cdot$ Eugene V. Beresin $^{4}$ • \\ Alan K. Louie ${ }^{5}$ - Laura Weiss Roberts ${ }^{5}$
}

Received: 9 March 2015 / Accepted: 17 March 2015 /Published online: 9 April 2015

(C) Academic Psychiatry 2015

The article by Robitz and colleagues [1] in this issue of Academic Psychiatry describes an entirely medical student-driven educational project, the Women Leading Healthy Change (WLHC). In this program, medical students at the University of Cincinnati partnered with a community organization to "educate and empower women who have survived commercial sexual exploitation, homelessness, substance dependence and mental illness" [1]. Their program was created with two missions: to empower sex workers with co-occurring mental illness and substance dependence and to teach medical students. As the authors point out, sex workers who live with addictions and mental illness (and, we add, even those without addiction and mental illness) are not typically viewed as potential educators of medical trainees. Nevertheless, individual medical students involved in this program perceived it as a great educational experience, which enabled them to deal with complicated and sensitive topics, made them more confident about taking a history related to underserved individuals who come from vulnerable populations, shifted their perspective toward women living with mental illness and substance abuse, and helped them "to recognize the humanness of us all" [1].

Decades ago, Erving Goffman [2] articulated the relationship between stigma and the "spoiled identity" of people who are marginalized and misunderstood in society. As Sartorius

Richard Balon

rbalon@wayne.edu

\footnotetext{
Wayne State University, Detroit, MI, USA

Dalhousie University, Halifax, NS, Canada

Baylor College of Medicine, Houston, TX, USA

4 Harvard Medical School, Boston, MA, USA

5 Stanford University, Stanford, CA, USA
}

[3] wrote, "the stigma attached to mental illness is the main obstacle to the provision of care for people with this disorder" (p. 810). He added that stigma also leads to discrimination in providing services for physical illness in those who are mentally ill. Finally, he reminded us that many people contribute to the development and reinforcement of stigma of mental illness, including health care workers: "Psychiatrists and mental health personnel are no exception in this general unawareness of how their behaviour contributes to stigma" ([3], p. 810).

Sex workers experience a similar occupational stigma with similar consequences. In a Canadian study of 252 sex workers [4], occupational sex worker stigma was independently associated with an elevated likelihood of experiencing barriers to health care access. Sex workers do not only face stigma and barriers in access to health care but may also face violence by their clients and police, as noted by Reza-Paul and colleagues from India [5]. The occupational stigma could be also complicated by additional stigma of HIV status [6, 7]. Bikmukhametov and colleagues [6] suggested that HIVpositive patients are often seen with bias and prejudice, especially if they are members of marginalized subgroups such as commercial sex workers and injection drug users. In their study of Russian medical students, they measured students' willingness to provide medical care to nine hypothetical categories of patients, four who were HIV negative (a physician, an injection drug user, a female commercial sex worker, and a man having sex with men) and five who were HIV positive (a physician, a recipient of a blood transfusion, an injection drug user, a female commercial sex worker, and a man having sex with men). The reluctance to provide health care was strongest in three groups: preclinical-level female students, clinicallevel female students, and clinical-level male students. Preclinical-level male students were more reluctant to provide health care to men having sex with men (both HIV negative and positive). In a similar study by Chan and colleagues [7], 
Chinese medical students were presented four clinical vignettes describing a hypothetical male. The vignettes were identical except for the presence of diagnosis (AIDS, leukemia, or no disease) and co-characteristic (injection drug user, commercial sex worker, commercial blood donation, blood transfusion, or no co-characteristic). After reading each vignette, students completed a measure of social distance that assessed the level of stigma. Interestingly, the most stigmatized scenario was an injection drug user without an accompanying disease, followed by an injection drug user with AIDS, a commercial sex worker without disease, and a commercial sex worker with AIDS.

Sex workers suffering from mental illness, possibly abusing substances, and possibly suffering from HIV-related illness could thus face multiple layers of stigmatizing prejudicial attitudes. These attitudes may create reluctance to provide health care to these individuals with overlapping sources of vulnerability and health needs. In the study by Bikmukhametov and colleagues [6], the majority (72\%) of female medical students were hesitant to provide care for HIVpositive sex workers. In an early study performed by Carter and Roberts [8], nearly all of the medical students at the University of Chicago and at the University of New Mexico endorsed the professional obligation to care for "all" patients, but fewer expressed willingness to provide care for HIVpositive patients, and significantly, fewer were willing if colleagues were aware of it. In Robitz and colleagues' article [1], most women reporting mental illness $(61 \%)$ had a history of sexual abuse as children (75\%), and almost half had a nonpsychiatric general medical diagnosis. Gu et al. [9] recently studied female injection drug users who were also sex workers and found that "self-stigma," or internalization of prejudicial attitudes and beliefs, was associated with depression, suicidal ideation, and suicide attempts. Dinos [10] outlined evidence that membership of a stigmatized group in and of itself (i.e., not based on mental illness) is often itself a risk factor for developing mental health problems. He examined the experiences of African-American people and minority ethnic and lesbian, gay, and bisexual groups to explore how stigma can "create" more stigma. Membership within such multiple groups is accompanied by several factors that can contribute to such compound stigma and, in turn, reduce economic and life opportunities, limit relationships and social support, delay seeking help, subvert funding for treatment, and diminish selfesteem, to name a few possible consequences.

Clearly, stigma is not a simple construct, and more often than not, people have the experience of being members of several stigmatized groups. There may well be and probably are other instances of stigma adding to and complicating stigma, such as in cases of mentally ill patients abusing substances, mental illness in obese patients, mental illness in incarcerated prisoners, or mental illness in parents with histories of child abuse or neglect.
Stigma of mental illness among medical students is prevalent and has been documented in numerous studies (e.g., [11-13]). Stigma against psychiatry as a profession is still prevalent in medicine and, no doubt, has an impact on psychiatry trainees and on the likelihood of learners pursuing psychiatry as a career. In a recent study of psychiatry trainees in Belgium [14], although $75 \%$ recalled hearing denigrating or humiliating comments about the profession more than once, only $1.3 \%$ recalled stigma as a topic during training.

We know little about the prevalence of stigma toward sex workers. Some studies $[6,7]$ suggest that this stigma is fairly prevalent. The state of the literature would suggest a significant gap in understanding the propagation of stigma within medical education, especially as it pertains to the many and varied groups who are stigmatized.

Programs to reduce stigma of mental illness have been introduced the European Union [1], and a program against stigma and discrimination because of schizophrenia was implemented in more than 20 countries worldwide [1]. Multiple programs to reduce the stigma of mental illness in undergraduate medical education $[10,11]$ or among medical students [12] have been described. Most of these reports evaluated impact of "interventions" such as clerkship in psychiatry or specialized courses. Several studies by O'Reilly and colleagues (e.g., $[15,16])$ examined yet another way to address reducing the stigma of mental illness mostly among pharmacy students - providing the students the opportunity to have contact with consumers with a mental illness in a safe educational setting. They reported that such contact led to decreases in stigma. They felt that sharing personal stories about mental illness was a powerful tool to decrease the stigma of mental illness and may be an important aspect of a person's recovery from mental illness. In a similar vein, Happell et al. [17] compared a mental health nursing course delivered traditionally to one delivered by an instructor with lived experience with mental illness and mental health service use. Impressively, the group that learned from the instructor with lived experience showed increased intention to pursue mental health nursing as a career and decreased negative stereotypes about mental illness; these findings were not observed in the group in the traditional course. In another study, O'Reilly and colleagues [18] found out that low level of mental illness stigma and high levels of schizophrenia "literacy" were associated with pharmacists being more willing to provide medication counseling and identify drug-related problems for patients with schizophrenia.

Reports of Robitz and colleagues [1], O'Reilly and colleagues [13], and Happel and colleagues [17] all suggest that the impact of contact with the stories of people suffering from medical and mental illness in an educational setting is very powerful and beneficial to all parties. Anecdotal accounts of educational efforts involving sex workers suggest that direct educational contact may be beneficial to remove the barriers to 
access to health care for these workers. We are not aware of studies other than that of Robitz et al. [1] on the impact of contact of persons with multiple layers of stigma or stigma within stigma - mental illness, substance abuse, and commercial sex work - on medical students and their education, on other professionals, and on patients themselves and their access to health care. Robitz et al. [1], as the creators of this program, and all their participants should be praised for the introduction of this innovative venture intended to help the most vulnerable, people with stigma within stigma. Their program is an example of using community-based partners from a vulnerable population as teachers. It is our hope that this effort will increase the empathy and concern of early-career physicians for this special patient group.

Finally, at a broad public health level, anti-stigma education around mental illness still has a long way to go and must, as Dinos [10] suggests, evolve to show the public the complex interaction between different forms and origins of stigma. Self-reflection and exploration of one's biases surrounding mental illness and other areas that surround stigma, such as race, using tools such as the Implicit Association Test [19] may help. The anti-stigma education should also include understanding of how media constructs and perpetuates stigmatizing depictions [20]. We definitely need to learn more about the complexities of how various factors (such as mental illness, sex workers, and substance abuse) might interact and contribute to stigmatizing attitudes. Tackling the potentially compounding effects of stigma added to stigma will require a robust approach at multiple levels - from the individual health care provider to the health professions and broader society.

Perhaps most importantly, as a matter of social justice, educational efforts to address stigma should be informed by the experience of those with lived experience, namely, giving voice to and valuing the perspectives of stigmatized people themselves. Robitz et al. [1] created such an opportunity, one that should remind us that if medical education is going to deliver the patient-centered care we promise, it will entail greater humanism in medicine. This humanism may be inspired and advanced through our greater understanding of the stories of those we care for and collaborate with in clinical education.

Disclosure On behalf of all authors, the corresponding author states that there is no conflict of interest.

\section{References}

1. Robitz R, Morrison L, Ventura A, Melton MC, Bennett A. Sex workers as medical student educators. Acad Psychiatry. 2015. doi: 10.1007/s40596-014-0214-8.
2. Goffman E. Stigma: notes on the management of spoiled identity. Englewood Cliffs: Prentice-Hall; 1963.

3. Sartorius N. Stigma and mental health. Lancet. 2007;370:810-1.

4. Lazarus L, Deering KN, Nabess R, Gibson K, Tyndall MW, Shannon K. Occupational stigma as a primary barrier to health care for street-based sex workers in Canada. Cult Health Sex. 2012;14: 139-50.

5. Reza-Paul S, Lorway R, O’Brien N, Lazarus L, Jain J, Bhagya M, et al. Sex worker-led structural interventions in India: a case study on addressing violence in HIV prevention through the Ashodaya Samithi collective in Mysore. Indian J Med Res. 2012;135:98-106.

6. Bikmukhametov DA, Anokhin VA, Vinogradova AN, Triner WR, McNutt LA. Bias in medicine: a survey of medical student attitudes towards HIV-positive and marginalized patients in Russia, 2010. J Int AIDS Soc. 2012;15:17372.

7. Chan KY, Yang Y, Zhang KL, Reidpath DD. Disentangling the stigma of HIV/AIDS from the stigmas of drug use, commercial sex and commercial blood donation - a factorial survey of medical students in China. BMC Public Health. 2007;7:280.

8. Carter DD, Roberts LW. Medical students' attitudes toward patients with HIV infection: a comparison study of 169 first-year students at the University of Chicago and the University of New Mexico. J Gay Lesbian Med Assoc. 1997;1:209-26.

9. Gu J, Lau JT, Li M, Li H, Gao Q, Feng X, et al. Socio-ecological factors associated with depression, suicidal ideation and suicidal attempt among female injection drug users who are sex workers in China. Drug Alcohol Depend. 2014;144:102-10.

10. Dinos S. Stigma creating stigma: a vicious circle. Psychiatr Bull. 2014;38:145-7.

11. Papish A, Kassam A, Modgill G, Vaz G, Zanussi L, Patten S. Reducing the stigma of mental illness in undergraduate medical education: a randomized controlled trial. BMC Med Educ. 2013;13:141.

12. Shen Y, Dong H, Fan X, Zhang Z, Li L, Lv H, et al. What can the medical education do for eliminating stigma and discrimination associated with mental illness among future doctors? Effect of clerkship training on Chinese students' attitudes. Int J Psychiatry Med. 2014;47:241-54.

13. Aggarwal AK, Thompson M, Falik R, Shaw A, O'Sullivan P, Lowenstein DH. Mental illness among us: a new curriculum to reduce mental illness stigma among medical students. Acad Psychiatry. 2013;37:385-91.

14. Catthoor K, Hutsebaut J, Schrijvers D, De Hert M, Peuskens J, Sabbe B. Preliminary study of associative stigma among trainee psychiatrists in Flanders, Belgium. World J Psychiatry. 2014;4: 62-8.

15. O'Reilly CL, Bell JS, Chen TF. Mental health consumers and caregivers as instructors for health professional students: a qualitative study. Soc Psychiatry Psychiatr Epidemiol. 2012;47:607-13.

16. O'Reilly CL, Bell JS, Chen TF. Consumer-led mental health education for pharmacy students. Am J Pharm Educ. 2010;74:167.

17. Happell B, Byrne L, Platania-Phung C, Harris S, Bradshaw J, Davies J. Lived-experience participation in nurse education: reducing stigma and enhancing popularity. Int J Ment Health Nurs. 2014;23:427-34.

18. O'Reilly CL, Bell JS, Kelly PJ, Chen TF. Exploring the relationship between mental health stigma, knowledge and provision of pharmacy services for consumers with schizophrenia. Res Soc Adm Pharm. 2013. doi:10.1016/j.sapharm.2013.04.006.

19. Project Implicit. Implicit Association Test. 2011. Available at http:// implicit.harvard.edu/implicit/. Last accessed 9 Mar 2015.

20. Nairn R, Coverdale S, Coverdale J. A framework for understanding media depictions of mental illness. Acad Psychiatry. 2011;35:202-6. 